\title{
Angiogenesis and schistosomiasis
}

\author{
Zilton A Andrade/ ${ }^{+}$, Thaynã Souto Santana \\ Laboratório de Patologia Experimental, Centro de Pesquisas Gonçalo Moniz-Fiocruz, Rua Valdemar Falcão 121, \\ 40295-001 Salvador, BA, Brasil
}

\begin{abstract}
Angiogenesis has been recognised as a precursor of fibrosis in several pathologic conditions. Its participation has been demonstrated in schistosomiasis, both during periovular granuloma formation and in the genesis of schistosomal periportal fibrosis. Paradoxically, proliferation of new blood vessels, accompanied by production of vascular-endothelial growth factor, appeared prominent during fibrosis regression months after curative treatment of schistosomiasis. Thus, angiogenesis in schistosomiasis seems to have a two-way mode of action, participating both in fibrogenesis and in fibrosis degradation. Morphological observations presented here are in keeping with the possibility that, in the first case, angiogenesis allows pericytes to come in great numbers to the site of lesions and be detached from capillary walls and transformed into myofibroblasts, which are important extra-cellular matrix forming cells. During post-curative fibrosis regression, actin-containing pericytes appeared at various foci of tissue remodelling, especially at sites of repair of vascular lesions. The molecular and cell factors involved in both situations seem to be important subjects in need of further investigations and the schistosomiasis model certainly will be of great avail in this regard.
\end{abstract}

Key words: Schistosoma mansoni - angiogenesis - hepatic fibrosis - pericytes - remodelling

Several studies have called attention to the fact that angiogenesis, the proliferation of numerous blood capillaries from pre-existing ones, precedes most types of hepatic fibrosis (Rosmorduc et al. 1999, Medina et al. 2005, Souza et al. 2006b). Recognising the importance of such reports, a recent editorial in a hepatology journal has suggested that anti-angiogenic drugs may soon be in use for the treatment of chronic liver diseases (Lai \& Adams 2005). This recent emphasis on the important role played by angiogenesis in fibrosis is probably related to the recognition of new factors and mechanisms in this field, but does not represent a novelty in the pathology of hepatic fibrosis. Considering whether hepatic fibrosis may sometimes originate from collapse of the supporting framework of the liver after massive parenchymal necrosis, McGee and Patrick (1972) emphasised that, even in such a special situation, hepatic fibrosis always results from granular tissue formation.

Our interest in the subject of angiogenesis and fibrosis started during experimental studies of hepatic fibrosis induced in rats by infection with the nematode Capillaria hepatica (Ferreira \& Andrade 1993, Andrade et al. 2005). It was then observed that $100 \%$ of the infected animals developed a peculiar type of septal fibrosis of the liver that started around the 15th day post-inoculation in portal spaces, connected portal to portal spaces and extended to the entire liver by the 20-25th day. The regularity of fibrosis development in this model allowed

+ Corresponding author: zilton@bahia.fiocruz.br

Received 18 June 2009

Accepted 9 October 2009 for the observation of its earliest stages. Then, sequential histological examination clearly indicated that angiogenesis preceded collagen synthesis during early septa formation (Souza et al. 2006a).

This process of fibrosis formation from granular tissue can occur anywhere in the living organism and represents a basic feature of general pathology. In the liver, it is thought to be dependent on a special cell-type, the parasinusoidal stellate cell, which is a pericyte, although with the peculiar feature of being able to store lipids and vitamin A (Friedman 2008). This peculiarity has facilitated its isolation from liver homogenates. Pure cell cultures are obtained after centrifugation in gradient medium, which is then followed by numerous in vitro studies. These important studies have lead to the impression that parasinusoidal stellate cells are the main, if not the sole, cell factor responsible for hepatic fibrosis production. However, recent studies on the relationship between angiogenesis and fibrosis are confirming the principle that hepatic fibrosis can originate from different structures in the liver, involving blood vessels and their associated cells and, of course, the hepatic sinusoids and their parasinusoidal stellate cells (Cassiman \& Roskams 2002).

Indeed, the classical morphological example of fibrosis formation in any organ follows the morphology and significance represented by the classical process of wound healing by second intention, in which the fundamental process of vascular proliferation from pre-existing blood vessels (angiogenesis) is prominent. An early explanation found in old textbooks of pathology suggested that the sprouting of new capillaries served to bring nutrients and oxygen to new repair tissue being formed by the resident fibroblasts and myofibroblasts. For some time, it passed unnoticed that capillaries are formed by a peculiar type of peripherally located cell, the pericyte. Although pericytes were described more than a century 
ago (Bergers \& Song 2005), their multiple and important functions are only now being recognised and explored (Lee et al. 2007). Being an actin-containing cell located within the capillary basement membrane, it was thought to be responsible for the contractility of the capillary wall and assumed that their single role was the regulation of the flux of blood. However, it is more than evident that pericytes can become detached from the capillary wall and then assume the phenotype and function of myofibroblasts inside the connective tissue (Fig. 1). Thus, the relationship between angiogenesis and fibrosis becomes obvious, since myofibroblasts have been demonstrated to be the most important cellular element in fibrogenesis (Cassiman et al. 2002, Medrado et al. 2008).

Angiogenesis and schistosomiasis - Angiogenesis plays a complex and extraordinary role in schistosomiasis. This statement may sound paradoxical, since schistosomes are intravascular parasites that cause lesions by destroying blood vessels, especially within the liver, as is the case with Schistosoma mansoni (Andrade 2004). The schistosome ova are deposited by the female worms inside blood vessels and are carried by the blood stream until they are retained within the smallest branches, where they become impacted and cause damage by means of their lytic and antigenic miracidial secretions. Thus, the endothelium of the capillaries is situated at the front line and is subjected to the initial damage and reaction in schistosomiasis. Lenzi et al. (1988) demonstrated that endothelial cells, immunohistochemically identified by factor-VIII, are present in great numbers within schistosomal periovular granulomas. They suggested that endothelial cells play a fundamental role, not only in granuloma formation, but in the pathogenesis of schistosomiasis in general. Additionally, studies in vitro have revealed that soluble egg antigens induce the proliferation of endothelial cells (Freedman \& Ottesen 1988) and also upregulate vascular endothelial growth factor (VEGF) and angiogenesis (Loeffler et al. 2002). Furthermore, studies with a combination of morphological techniques by Baptista and Andrade (2005) revealed that angiogenesis plays a dynamic and fundamental role during schistosomal periovular granuloma in vivo. Vascular proliferation can be noted throughout the early periovular granuloma, but soon are gradually displaced toward its periphery and end up forming a vascular collar around

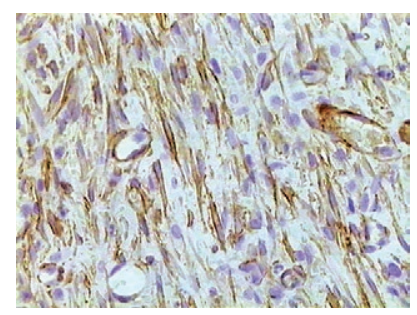

Fig. 1: granulation tissue formed one week following an experimental wound inflicted on the dorsal skin of a normal rat. Staining for actin marks both interstitial fusiform myofibroblasts and peri-capillary pericytes, suggesting a common origin for these cell-types involved in wound healing. Actin (DAKO, clone 1A4). 400X. the periovular granuloma, while its centre may appear almost avascular. When there is fusion of several granulomas, as occurs in periportal fibrosis during heavy infection both in man and in mouse, the proliferating small blood vessels appear prominent in inter-granulomatous tissue and the fibrous tissue assumes an angiomatoid appearance. In spite of this evidence of vascular proliferation, many vessels are indeed destroyed. In severe infections, the peripheral portal vasculature becomes progressively amputated, resulting in increasing intraportal pressure, thus creating conditions that cause the new eggs to be impacted and to cause damage to the fine collateral veins emerging from larger and larger portal vessels. Thus, portal vein branches of different calibres can become partially or totally occluded (Fig. 2). The vascular plastic casts obtained from the liver at such late stages of infection have revealed variable degrees of intrahepatic portal vein obstruction, coupled with preservation of the hepatic veins and hyperplasia and hypertrophy of the hepatic artery (Andrade \& Cheever 1971, Silva et al. 2006).

Angiogenesis following curative treatment of schistosomiasis - Andrade et al. (2005) demonstrated that mice with a four month-50 cercaria infection presented a poor portal vasculature with a "dry-tree" appearance seen in vascular casts obtained from portal vein system injections with vinyl-acetate (Fig. 2A). Four months after curative treatment, application of the same vascular technique revealed a completely different picture (Fig. 2B), with numerous small branches of vessels sprouting from and around the larger portal segments. These findings give the impression at first inspection that the post-chemotherapy increase of the vasculature resulted from pressure dilatation into an almost empty capillary bed after repair and dilatation of the larger portal vessels. However, a close microscopic observation revealed that there were several focal areas of vascular proliferation associated with the presence of a positive staining for the VEGF, plus the appearance of many actin-staining proliferating cells. Therefore, a process of angiogenesis was occurring during the curative phase of hepatic schistosomiasis.

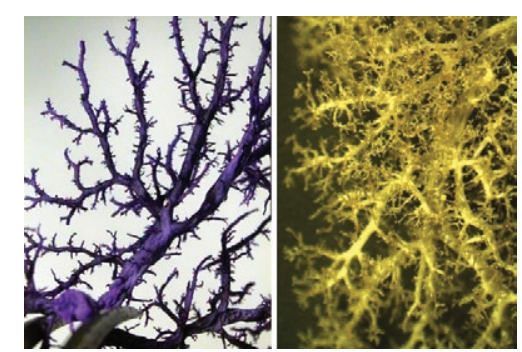

Fig. 2A: plastic vinyl cast obtained from the portal vein of a mouse with a four month-old, 50 cercaria-Schistosoma mansoni infection. A paucity of the vascular ramifications and a hairy aspect along the larger branches of the portal vessels can be observed; B: the fine peripheral vascular branching, which was lacking in the previous cast obtained from an infected animal (2A), re-appears in this mouse six months after treatment of the schistosomal infection with oxamniquine-praziquantel. 
This brings about the interesting question whether angiogenesis may function along a two-way tract, being important during fibrosis production as well as during fibrosis regression.

As a matter of fact, when a patient with hepatosplenic schistosomiasis is subjected to curative treatment, he will usually progress to a cure months or years later. Data from human and experimental studies have revealed that in such situations hepatic fibrosis regresses, accompanied by a reduction in the spleen size, a drop in portal pressure and a reduction of oesophageal varices (Bina \& Prata 1983, Dietze \& Prata 1986, Homeida et al. 1988, Mohamed-Ali et al. 1991, Richter 2003). In that way, it may be assumed that not only has hepatic fibrosis been degraded and reduced, but that a considerable degree of intra-hepatic vascular remodelling has also occurred.

Angiogenesis and remodelling in schistosomiasis One may wonder why angiogenesis appears to be an important feature both in fibrosis formation and in fibrosis degradation. Or, in other words, what is present in angiogenesis that may interfere with both processes? In the first case, the importance of the pericyte/myofibroblast axis is evident. For the second alternative, there are new data indicating that the pericyte/endothelium axis may be involved in remodelling, at least with the damaged vasculature. As recently stated by Lee et al. (2007), "for a long time, the existence and role of pericytes were neglected, but during recent years these cells have gained increasing attention, not only as contractile cells, but also as obligatory regulators of vascular development, stabilization, maturation and remodeling".

The findings observed after specific chemotherapy of schistosomiasis in mice, described by Andrade et al. (2005) and presently being amplified (some new data be- ing included in this report), are strongly indicative of the role played by a capillary-associated, actin-containing cell in remodelling of the extra-cellular matrix and its associated vascular lesions. Medium-sized portal veins, which were frequently occluded by chronic inflammatory tissue in liver biopsies from mice with a four monthold $S$. mansoni infection, were no longer observed in autopsy materials from the same animals four months after treatment with oxamniquine-praziquantel (Andrade et al. 2005). Other similar observations indicative of vascular remodelling were also observed. These included endothelial proliferation with the formation of new lumina inside the primitive vessel, focal disappearance of elastic tissue in areas of sub-endothelial elastosis and degradation of peri-vascular fibrosis. These studies were recently resumed to include material obtained six months after treatment. This time, immunohistochemical methods for demonstrating VEGF and smooth-muscle actin were also included. The association of vascular proliferation with VEGF and the presence of actin-positive cells were quite evident in the lesions (Fig. 3). Vessels exhibiting sub-endothelial elastic hyperplasia (elastosis) frequently showed focal areas of elastic dissolution. Sometimes these lytic areas appeared as oedematous young connective tissue with proliferated capillaries (Fig. 4).

Lesions involving larger branches of the intra-hepatic portal vein, which appeared occluded by a mixture of granulation tissue and focal areas of necrosis, were attributed to the death of adult worms ("dead-worm lesions"). Such lesions were eventually detected in liver sections taken six months after curative treatment of schistosomiasis (Fig. 5). They reveal no tendency toward fibrous repair, but rather one of remodelling toward the formation of numerous new vascular channels.
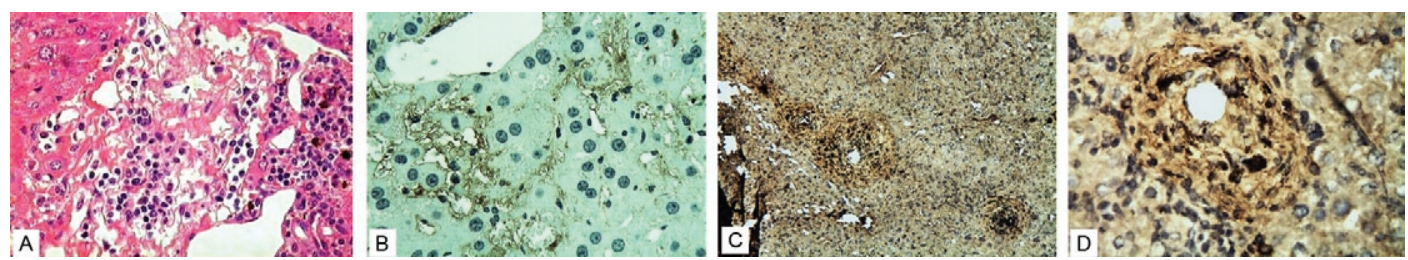

Fig. 3A: the spongy appearance of a well vascularized area of fibrosis regression in the liver of a schistosome infected mouse, four months after specific treatment. H\&E, 200X; B: the same area shown in A reveals the presence of vascular endothelial growth factor (VEGF) (Santa Cruz Biotechnology) in relation to the zones of vascular proliferation. Immuno-histochemistry, 200X; C, D: the presence of VEGF is evident inside the involuting periovular granulomas, as observed six months after curative treatment of murine schistosomiasis. Immunohistochemistry: C: 200X; D: 400X.
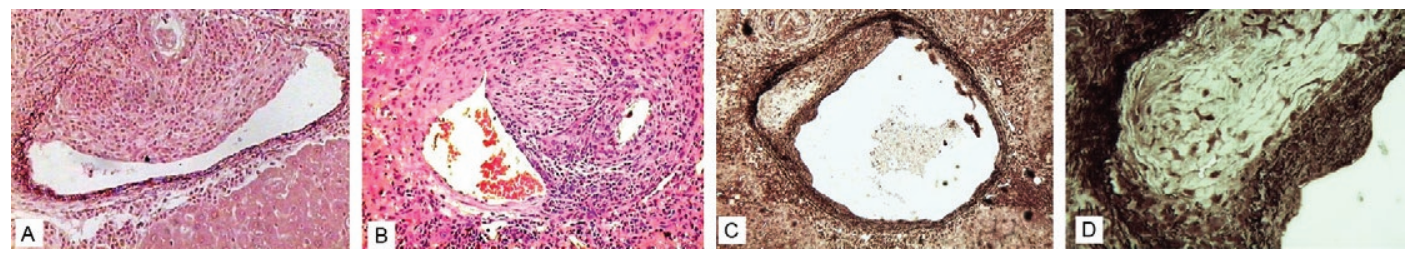

Fig. 4: schistosomal vascular lesions involving medium-sized branches of the portal vein. A: four month-old non-treated schistosomal infection showing a large granuloma formed around an egg impacted at the opening of a small collateral branch, causing partial destruction of the vascular wall. H\&E, 400X; B: a similar lesion is observed four months after curative treatment. Evidences of remodeling can be seen in the polarization of fusiform matrix cells and the opening of small vascular lumina within the reparative tissue. H\&E, 400X; C, D: sub-intimal repair in a medium-sized portal vein branch, seen in a schistosome-infected mouse six months after curative treatment. There are elastic fibers lysis and differentiation of small capillaries. Orcein stain 400X. 


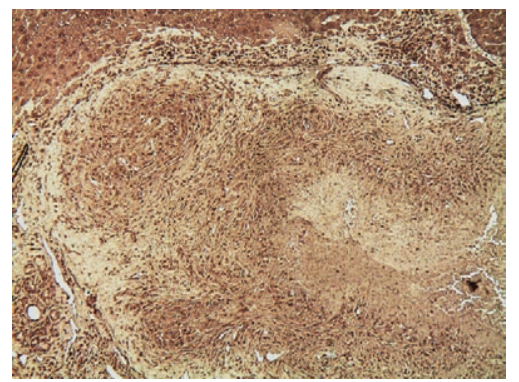

Fig 5: a large portal vein branch appears occluded by several foci of vascular proliferation (angiogenesis) formed around an old necrotic area (delimited by the arrows). Orcein stain, 400X.

Angiogenesis, pericytes and remodelling - The material obtained from treated schistosomiasis of the mouse offers a series of evidence that hepatic schistosomal lesions undergo considerable remodelling with time. Obstructive vascular lesions are partially or completely repaired; the excess extracellular matrix undergoes regression at the same time numerous small blood vessels are sprouting in several directions. When larger portal veins are obstructed by necro-inflammatory tissue, probably originating from the death of adult worms in situ, there later appeared several foci of vascular proliferation (angiogenesis) instead of classical repair changes, with fibrous replacement as observed in ordinary vascular thrombosis. Probably the same cells, endothelial cells and pericytes, are at play in both instances; however, the local factors generated in both situations are probably different. Considerable further studies seem necessary to clarify the molecular mechanisms involved. However, what is clear at the present moment is that the endothelium-pericyte axis is involved and that accentuates the importance of angiogenesis in both fibrosis formation and remodelling of the lesions following curative treatment in schistosomiasis and calls attention to the plasticity of pericytes, considered a primitive cell, with the potential of a true stem cell.

\section{REFERENCES}

Andrade ZA 2004. Schistosomal hepatopathy. Mem Inst Oswaldo Cruz 99 (Suppl. I): 51-57.

Andrade ZA, Assis BCA, Souza MM 2005. Capillaria hepatica: papel em patologia humana e potencial como modelo experimental. In JR Coura, Dinâmica das doenças infecciosas e parasitárias, Editora Guanabara-Koogan, Rio de Janeiro, p. 1121-1132.

Andrade ZA, Cheever AW 1971. Alterations of the intrahepatic vasculature in hepatosplenic schistosomiasis mansoni. Am J Trop Med Hyg 20: 425-432.

Baptista AP, Andrade ZA 2005. Angiogenesis and schistosomal granuloma formation. Mem Inst Oswaldo Cruz 100: 183-185.

Bergers G, Song S 2005. The role of pericytes in blood-vessel formation and maintenance. Neuro Oncol 7: 452-464.

Bina JC, Prata A 1983. A regressão da hepatoesplenomegalia pelo tratamento específico da esquistossomose. Rev Soc Bras Med Trop 16: $213-218$.

Cassiman D, Libbrecht L, Desmet V, Denef C, Roskams T 2002. Hepatic stellate cell/myofibroblast subpopulations in fibrotic human and rat livers. J Hepatol 36: 200-209.
Cassiman D, Roskams T 2002. Beauty is in the eye of the beholder: emerging concepts and pitfalls in hepatic stellate cell research. J Hepatol 37: 527-535.

Dietze R, Prata A 1986. Rate of reversion of hepatosplenic schistosomiasis after specific therapy. Rev Soc Bras Med Trop 19: 69-73.

Ferreira LA, Andrade ZA 1993. Capillaria hepatica: a cause of septal fibrosis of the liver. Mem Inst Oswaldo Cruz 88: 441-447.

Freedman DO, Ottesen EA 1988. Eggs of Schistosoma mansoni stimulate endothelial cell proliferation in vitro. J Infect Dis 158: 556-562.

Friedman SL 2008. Hepatic stellate cells: protean, multifunctional and enigmatic cells of the liver. Physiol Rev 88: 125-172.

Homeida M, Ahmed S, Dafalla A, Suliman S, Eltom I, Nash T, Bennett JL 1988. Morbidity associated with Schistosoma mansoni infection as determined by ultrasound: a study in Gezira, Sudan. Am J Trop Med Hyg 39: 196-201.

Lai WK, Adams DH 2005. Angiogenesis and chronic inflammation; the potential for novel therapeutic approaches in chronic liver disease. J Hepatol 42: 7-11.

Lee JS, Semela D, Iredale J, Shah VH 2007. Sinusoidal remodeling and angiogenesis: a new function for the liver-specific pericyte? Hepatology 45: 817-825.

Lenzi HL, Sobral AC, Lenzi JA 1988. Participation of endothelial cells in murine schistosomiasis. Braz J Med Biol Res 21: 999-1003.

Loeffler DA, Lundy SK, Singh KP, Gerard HC, Hudson AP, Boros DL 2002. Soluble egg antigens from Schistosoma mansoni induce angiogenesis-related processes by up-regulating vascular endothelial growth factor in human endothelial cells. J Infect Dis 185: 1650-1656.

McGee JO, Patrick RS 1972. The role of perisinusoidal cells in hepatic fibrogenesis. An electron microscopic study of acute carbon tetrachloride liver injury. Lab Invest 26: 429-440.

Medina J, Sanz-Cameno P, García-Buey L, Martín-Vílchez S, LópezCabrera M, Moreno-Otero R 2005. Evidence of angiogenesis in primary biliary cirrhosis: an immunohistochemical descriptive study. J Hepatol 42: 124-131.

Medrado AP, Soares AP, Santos ET, Reis SR, Andrade ZA 2008. Influence of laser photobiomodulation upon connective tissue remodeling during wound healing. J Photochem Photobiol B 92: 144-152.

Mohamed-Ali Q, Doehring-Schwerdtfeger E, Abdel-Rahim IM, Schlake J, Kardorff R, Franke D, Kaiser C, Elsheikh M, Abdalla M, Schafer P, Ehrich JHH 1991. Ultrasonographical investigation of periportal fibrosis in children with Schistosoma mansoni infection: reversibility of morbidity seven months after treatment with praziquantel. Am J Trop Med Hyg 44: 444-451.

Richter J 2003. The impact of chemotherapy on morbidity due to schistosomiasis. Acta Trop 86: 161-183.

Rosmorduc O, Wendum D, Corpechot C, Galy B, Sebbagh N, Raleigh J, Housset C, Poupon R 1999. Hepatocellular hypoxia-induced vascular endothelial growth factor expression and angiogenesis in experimental biliary cirrhosis. Am J Pathol 155: 1065-1073.

Silva LM, Ribeiro-Dos-Santos R, Soares MB, Andrade ZA 2006. Characterization of the vascular changes in schistosomal portal (pipestem) fibrosis of mice. Acta Trop 98: 34-42.

Souza M, Tolentino M Jr, Assis BC, de Oliveira Gonzalez AC, Maria Correia Silva T, Andrade ZA 2006a. Pathogenesis of septal fibrosis of the liver (an experimental study with a new model). Pathol Res Pract 202: 883-889.

Souza MM, Tolentino M Jr, Assis BC, de Oliveira Gonzalez AC, Silva TMC, Andrade ZA 2006b. Significance and fate of septal fibrosis of the liver. Hepatol Res 35: 31-36. 